\title{
Les constructions exceptives vues comme des listes paradigmatiques: à propos de la syntaxe de sauf, excepté, hormis... en français
}

\author{
Mohamed Galal ${ }^{1,2}$ et Sylvain Kahane ${ }^{1}$ \\ ${ }^{1}$ Laboratoire MoDyCo - UMR 7114, Université Paris Nanterre, 92001 Nanterre Cedex, France \\ ${ }^{2}$ Département du français, Faculté des Lettres, Université de Sohag, 82524 Sohag, Égypte \\ mohamed.moustafa@parisnanterre.fr, sylvain.kahane@parisnanterre.fr
}

\begin{abstract}
Résumé. Nous proposons dans cet article une description syntaxique des constructions exceptives (CE) en français qui tient compte de la diversité phénoménologique de ces constructions découlant de la diversité des marqueurs mis en jeu. Nous proposons une classification des CE en deux classes majeures à comportements syntaxiques distincts : les CEparadigmatiques qui s'apparentent, du point de vue syntaxique, à la coordination et les CE-hypotactiques qui relèvent au contraire de la subordination. Nous situerons notre analyse des marqueurs sauf, excepté, hormis, etc. dans le cadre des listes/entassements paradigmatiques, constructions dans lesquelles deux éléments occupent la même position syntaxique et dont le cas le plus connu est la coordination. Cette analyse s'éloigne de celle généralement associée à ces marqueurs dans les grammaires et les dictionnaires français qui les traitent comme des prépositions.
\end{abstract}

\begin{abstract}
Exceptive constructions considered as paradigmatic lists: on the syntax of sauf, excepté, hormis... in French. We propose in this article a syntactic description of exceptive constructions (EC) in French which takes into consideration the phenomenological diversity in this construction resulting from the variations of the markers involved. We suggest a classification of ECs into two major categories with dissimilar syntactic behaviors: the paradigmatic-ECs, which are syntactically related to coordination and the hypotactic-ECs, which are contrarily related to subordination. We will focus our analysis on the markers sauf, excepté, hormis, etc. and analyze them as particular case of paradigmatic lists/piles, in which two segments of the utterance pile up on the same syntactic position and whose most famous case is coordination. This analysis is different from the one generally associated with these markers in French grammars and dictionaries which consider them as prepositions.
\end{abstract}

\section{Introduction}

L'objectif de cet article est de proposer une description du fonctionnement syntaxique des constructions exceptives du type [X sauf $\mathrm{Y}]$ en français. Le phénomène de l'exception peut 
se définir comme un processus sémantique qui met en relation un ensemble $\mathrm{X}$ et un sousensemble Y au plan de la quantification (cf. Léard et Marengo, 2006). On attribue une propriété à $\mathrm{X}$, puis on nie l'attribution de cette propriété à $\mathrm{Y}$ (voir figure 1). X et $\mathrm{Y}$ sont assujettis à certaines conditions (cf. Kleiber, 2005 ; Moltmann 1992 ; 1995) : (i) Y doit donner lieu à une prédication de polarité opposée de $\mathrm{X}$ (ii) $\mathrm{Y}$ doit être inclus dans $\mathrm{X}$, (iii) $\mathrm{X}$ et $\mathrm{Y}$ doivent être bien délimités, (iv) $\mathrm{X}$ doit être universel au niveau de la quantification ${ }^{1}$, (v) Y doit être toujours une quantité faible par rapport à X.

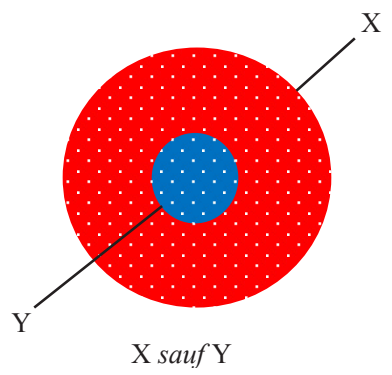

Fig. 1. Conditions sur $\mathrm{X}$ et $\mathrm{Y}$ pour une exception

Les constructions exceptives en français sont réalisées par les marqueurs sauf, excepté, hormis, sinon, etc. et les locutions prépositives à l'exception de, exception faite de, à l'exclusion de, exclusion faite de, en dehors de, (mis) à part, etc. La plupart de ces marqueurs sont polysémiques et présentent une variété d'emplois autre que l'exception comme la réserve (1a), l'addition (1b), la disjonction ${ }^{2}$ (1c), etc. et dont l'étude de la sémantique dépasse le cadre de cet article.
a. Sauf erreur de ma part, aucune annonce n'a été faite aujourd'hui concernant les victimes. (lemonde.fr)
b. En 2024, à part moi, on pourrait avoir trois autres Yoka : mon frère Victor qui aura 26 ans, mon frère Axel qui en aura 24 et ma petite sœur (Shannon) qui en aura 23. (leparisien.fr)
c. On sait qu'il va falloir désormais s'habituer au choc d'un événement planétaire chaque jour, sinon chaque semaine! (nouvelobs.com)

La construction exceptive est souvent analysée comme une construction binaire formée de deux structures prédicatives : une proposition principale et une proposition elliptique du prédicat que le marqueur met en relation (cf. Biardzka, 2011, Ruozzi, 2008). La proposition elliptique partage implicitement le prédicat de la proposition principale, mais avec une polarité inverse (2).

[L'exécutif contrôle tous les ressorts du pouvoir], [sauf le Parlement] $\rightarrow$ 'l'exécutif ne contrôle pas le Parlement'. (lemonde.fr)

Nous opterons pour le terme de construction exceptive (dorénavant $\mathrm{CE}$ ) pour désigner la construction en cause. La proposition principale est celle qui contient le constituant auquel s'applique l'exception et que nous appellerons l'antécédent exceptif ou simplement l'antécédent (dorénavant Ant). L'Ant sera souligné en rouge dans les exemples. Nous utiliserons le terme de marqueur exceptif (dorénavant ME) pour designer toutes les unités lexicales qui véhiculent l'exception (sauf dans l'exemple) et elles seront soulignées en bleu. L'élément exclu introduit par le ME sera désigné par le terme élément excepté (dorénavant $\mathrm{EE})$ et sera souligné en vert. Le syntagme formé du ME et son complément, l'EE, sera nommé le syntagme exceptif (dorénavant SE). La terminologie et les conventions sont illustrés en (3).

$$
\text { [L'exécutif contrôle [tous les ressorts du pouvoir] Ant, [[sauf] ME [le Parlement] EE] SE] CE. }
$$


L'exception a fait l'objet de plusieurs travaux en français (cf. Biardzka, 2011 ; Kleiber, 2005, Léard et Marengo, 2006 ; Moignet, 1959 ; Mourin, 1980 ; Roig et Rosier, 2012 Ruozzi, 2008), mais peu d'entre eux privilégient la syntaxe, à l'exception de Piot, 2005. Concernant la syntaxe de l'exception, on pourra consulter Ionescu, 2013 pour le romain ; Pérez-Jimenéz et Mareno-Quibén, 2012 pour l'espagnol ; Potsdam et Polinsky, 2017 pour le tahitien ; Sultan, 2016 pour l'arabe égyptien. Les propriétés sémantiques définitoires des $\mathrm{CE}$ ont quant à elles été discutées à de nombreuses reprises dans le cadre de la sémantique formelle, généralement à partir de l'anglais, en se fondant sur la théorie des quantifieurs généralisés (angl. Generalized Quantifier Theory): Crnič, 2016 ; von Fintel, 1993 ; Gajewski, 2008 ; García Álvarez, 2008 ; Hoeksema, 1987 ; 1995 ; Lappin, 1996 et Moltmann, $1992 ; 1995$.

À partir d'un corpus d'un millier d'exemples attestés, nous proposons une classification des CE en français en deux classes : les CE-paradigmatiques, qui s'apparentent, du point de vue syntaxique, à la coordination, et les CE-hypotactiques ${ }^{3}$, qui relèvent au contraire de la subordination. Cette typologie s'inspire de la taxonomie bien établie dans la littérature anglophone sur les CE, initialement proposée par Hoeksema (1987 ; 1995), entre "connected exceptives» et "free exceptives ${ }^{4}$, mais raffine aussi certaines notions de celle-ci (cf. section 4.4).

Nous proposons d'analyser les marqueurs sauf, excepté, hormis, etc. dans le cadre des entassements/listes paradigmatiques (cf. Blanche-Benveniste, 1990 ; Gerdes \& Kahane, 2009 ; 2015 ; Guénot, 2005 ; Kahane et Pietrandrea, 2012 ; Nølke, 1983) dans lesquelles deux éléments peuvent occuper la même position syntaxique et dont le cas le plus connu est la coordination. Cette analyse va à l'encontre de celle généralement associée à ces marqueurs dans les grammaires et les dictionnaires français qui les traitent comme des prépositions. En effet, nous considérons que sauf, excepté, hormis, dans leur emploi exceptif, n'ont pas les propriétés d'une préposition, mais plutôt celles d'une conjonction de coordination, puisque ces marqueurs peuvent être suivi, en plus du groupe nominal, d'un groupe prépositionnel (4a) ou d'une proposition adverbiale (4b). En plus, ils commutent avec une conjonction de coordination comme mais pas (5a) ou un adverbe paradigmatisant (cf. Nølke, 1983) comme même (5b).

(4) a. La grippe A décline partout en Europe, sauf en France. (lemonde.fr)

b. Marie-Lou est à pied d'œuvre tous les jours, sauf quand elle est en vadrouille. (leparisien.fr)

(5) a. La grippe A décline partout en Europe, mais pas en France.

b. La grippe A décline partout en Europe, même en France.

Cette perspective a été confortée par des arguments assez proches pour l'anglais par Harris (1982), Reinhart (1991) et García Álvarez (2008) ainsi que pour l'espagnol par Pérez-Jimenéz et Mareno-Quibén (2012) et pour l'arabe égyptien par Soltan (2016), qui concluent que les ME dans ces langues sont des conjonctions de coordination.

L'article se présente de la façon suivante : dans la section 2, nous présentons notre classification des constructions exceptives en CE-paradigmatiques et CE-hypotactiques et nous exposons les critères que nous avons adoptés pour le classement des données. Dans la section 3, nous proposons d'analyser les CE-paradigmatiques comme un cas particulier de listes paradigmatiques et nous discutons un certain nombre de propriétés qui distinguent, ou semblent distinguer, les CE-paradigmatiques de la coordination. Dans la section 4, nous examinons la partie du discours des ME. Nous exposons les quatre analyses existantes de ces éléments: préposition, adverbe, conjonction de subordination ou conjonction de coordination et nous fournissons des arguments supplémentaires en faveur de la coordination. Les conclusions de ce travail font l'objet de la section 5. 


\section{Les deux types de constructions exceptives en français}

Nous allons maintenant justifier le classement des $\mathrm{CE}$ en deux classes, les $C E$ paradigmatiques et les CE-hypotactiques. Les deux types sont canoniquement illustrés en (6).

(6) CE-paradigmatique a. Tous les partis, sauf l'UMP, veulent une "dose" de

CE-hypotactique b. Tous ces bâtiments, à l'exception du Cirque d'Hiver sur les grands boulevards, ont malheureusement disparu. (bief.org)

Les CE-paradigmatiques sont introduites par les marqueurs simples sauf, excepté, hormis, à part et sinon ${ }^{5}$; alors que les CE-hypotactiques sont régies par les locutions prépositives à l'exception de, exception faite de, à l'exclusion de, exclusion faite de, en dehors de, etc. (Voir le tableau 1 ci-dessous) ${ }^{6}$.

Tableau 1. Classification des ME

\begin{tabular}{|c|c|}
\hline Les CE-paradigmatiques & Les CE-hypotactiques \\
\hline sauf & àl'exception de \\
\hline excepté & exception faite de \\
\hline hormis & $\grave{a}$ l'exclusion de \\
\hline à part & exclusion faite de \\
\hline sinon & en dehors de \\
\hline
\end{tabular}

Nous allons voir dans la suite que les CE-paradigmatiques partagent plusieurs propriétés avec la coordination : (i) la place linéaire du SE, (ii) la catégorie syntaxique de l'EE et (iii) la possibilité ou non de coordonner le SE. Pour chacune de ces propriétés, les CEhypotactiques possèdent un comportement différent.

\subsection{La place linéaire du syntagme exceptif}

Dans les CE-paradigmatiques, le SE se place généralement en fin d'énoncé (7). Une place du SE en tête de l'énoncé (8a) ou inséré dans le SV (8b) ne sont pas possibles.

(7) a. Le feu détruisit tous les bâtiments, sauf l'église romane. (marieclauderando.free.fr)

b. Tous les lots ont trouvé preneur, excepté le Morandi. (lemonde.fr)

c. Les joueurs sont protégés par la bulle qui couvre toutes les parties du corps hormis le bas des jambes. (bubblebump.frweb)

d. Elles ne sont d'accord sur rien, à part sur l'urgence de remonter le moral de Françoise, leur mère $[\ldots]$. (allocine.fr)

e. Je suis stupéfait mais ne peux rien faire sinon prévenir mon collègue de l'université. (huffingtonpost.fr)

(8) a. *Sauf l'église romane, le feu détruisit tous les bâtiments.

b. *Le feu a, sauf l'église romane, détruit tous les bâtiments.

Cependant, le SE peut se placer en position contiguë à l'Ant, surtout avec l'Ant nominal (9) ; une position moins fréquente dans le cas des autres types de syntagme, mais qui est possible aussi (10).

(9) a. Tous les partis, sauf le PP, sont favorables au transfert des restes du dictateur hors du mausolée abritant les ossements de milliers de victimes de la guerre civile. (lemonde.fr)

b. Toutes les salles excepté l'auditorium bénéficient de la lumière du jour. (lieuxoutsita.free.fr)

c. Tous les prix, hormis celui du public qui sera remis dimanche, ont été décernés hier soir. (lamontagne.fr) 
d. Personne, à part ceux qui ont vécu cette situation, ne peut mesurer la pression inhérente à un match décisif dans une finale de Coupe Davis, qui plus est en étant favori, et à domicile. (eurosport.fr)

e. Rien, sinon le sens du défi, ne prédestinait cet ancien cadre supérieur à prendre les rênes en 2005 de Corona Etanchéité. (lemonde.fr)

(10) a. Les marchés boursiers à travers le monde, sauf en Europe, étaient généralement haussiers ce vendredi matin. (Corpus du français de Leipzig)

b. Partout, excepté en France, les courants de la figuration moderne de l'entre-deux-guerres ont connu un réexamen suivi d'une réévaluation. (bibliopolis.be)

c. Dans la totalité des régions métropolitaines, hormis en Bretagne, la gauche recule en nombre d'élus [...]. (lemonde.fr)

Notons que le syntagme introduit par sauf, excepté, hormis, (mis) à part peut se trouver à d'autres places, notamment en tête d'énoncé, dans d'autres constructions que l'exception (voir la section 2.5).

Les CE-hypotactiques ont une distribution plus libre que les CE-paradigmatiques. Elles permettent la position antéposée (11), l'adjonction à l'Ant (12), la parenthétisation entre l'auxiliaire et le participe (13) et la postposition (14).

(11) a. À l'exception de la CGT [...], tous les syndicats affichent [...] une positive attitude. (lemonde.fr)

b. En dehors de Khaled Ali, tous ces hommes étaient des piliers de l'ancien régime d'Hosni Moubarak. (lemonde.fr)

(12) a. Tous les départements, à l'exception de la Corse, sont touchés par l'épidémie de bronchiolite. (topsante.com)

b. La disponibilité de tous ces ordinateurs, exception faite de l'iMac Pro, est fixée à aujourd'hui. (macg.co)

(13) a. Pour obtenir le nouveau véhicule, les syndicats ont - à l'exception de la CGT accepté de consentir de nouveaux efforts [...]. (leparisien.fr)

b. Ces rencontres à l'étranger ont, à l'exclusion de la première session, à chaque fois accueilli François Fillon. (liberation.fr)

(14) a. Aucun de ces véhicules ne possède de carte grise, à l'exception de quatre d'entre eux [...]. (lefigaro.fr)

b. Personne ne le remarque, en dehors de Jaco. (dragonball-ultimate.com)

\subsection{La catégorie syntaxique de l'EE}

Il s'agit de tester à présent l'interaction entre les ME et les différents types de syntagmes avec lesquels ils peuvent être combinés. Les ME dans l'emploi paradigmatique, dont sauf est le représentant, peuvent s'adjoindre à une grande variété de constituants contrairement aux ME dans l'emploi hypotactique, dont à l'exception de est le prototype, qui ne peuvent être combinés qu'avec un substantif (15 vs 16). Comme le montrent les exemples en (15), sauf peut se combiner avec un syntagme nominal (15a), un syntagme verbal à l'infinitif (15b), un syntagme verbal introduit par une préposition (15c), mais aussi une phrase (15d), un adverbe (15e), un adjectif (15f) et un syntagme prépositionnel $(15 \mathrm{~g})$.

(15) a. Ce robot fait tout comme les chiens, sauf [les câlins]. SN (dailygeekshow.com)

b. On peut presque tout faire sans lumière sauf [écrire]. (Michel Serres)

$S V_{\text {inf }}$

c. Plus rien n'a d'importance sauf [de faire le maximum d'attractions]. SV prép+inf (lemonde.fr)

d. Manifestement, on a tout compris, sauf [ce que veut le peuple]. Pce que (lemonde.fr)

e. Les marchés publics chinois ont toujours été fermés aux étrangers, sauf SAdv [quand il s'agit d'obtenir des hautes technologies]. (lemonde.fr)

f. Les Français ne se livrent à aucun mouvement d'émeute sauf [sporadique SAdj et très isolé]. (hypnose-medium-jura.e-monsite.com) 
g. Le nombre de détenus baisse partout en Europe, sauf [en France]. SP (lemonde.fr)

(16) a. Ce robot fait tout comme les chiens, à l'exception des [câlins].

b. *On peut presque tout faire sans lumière à l'exception d' [écrire].

c. *Plus rien n'a d'importance à l'exception de [de faire le maximum d'attractions].

d. Manifestement, on a tout compris, à l'exception de [ce que veut le $\mathrm{P}_{\text {ce que }}$ peuple].

e. *Les marchés publics chinois ont toujours été fermés aux étrangers, à l'exception de [quand il s'agit d'obtenir des hautes technologies].

f. *Les Français ne se livrent à aucun mouvement d'émeute à l'exception de *SAdj [sporadique et très isolé].

g. *Le nombre de détenus baisse partout en Europe, à l'exception de [en *SP France].

\subsection{La possibilité ou non de coordonner le SE}

Les CE-paradigmatiques ne présentent pas la possibilité de se coordonner ${ }^{7}$ (17a vs b). Au contraire, les CE-hypotactiques offrent une telle possibilité. Dans le cas des locutions prépositives, la coordination se passe toujours en reprenant la préposition de (18).

(17) a. Ouvert tous les jours, sauf lundis, mardis et jours fériés. (lemonde.fr)

b. *Ouvert tous les jours, sauf lundis, sauf mardis et sauf jours fériés

(18) Les syndicats, à l'exception de FO et de la CFDT, manifestent mardi 12 septembre contre les ordonnances sur la réforme du droit du travail. (lemonde.fr)

\subsection{Remarques à propos de la contrainte sur la quantification universelle}

Selon plusieurs auteurs (cf. von Fintel, 1993 ; Hoeksema, 1987 ; Keiber, 2005 ; Lappin, 1996 ; Moltmann, 1995 ; Pérez-Jimenéz et Mareno-Quibén, 2012), les deux types de constructions imposent des contraintes sur le type de quantifieur présent dans l'Ant. Les CE-paradigmatiques doivent avoir pour Ant un SN contenant obligatoirement un quantifieur universel, soit positif tous, tout, tout le monde, etc. (19a), soit négatif aucun, rien, personne, etc. (19b), dont le SE sert à limiter la portée universelle. Les constructions dont l'antécédent comporte un quantifieur quasi-universel la plupart, la majorité, etc. (20a), non-universel quelques ou un quantifieur de masse beaucoup de et peu de (20b) sont prédites agrammaticales. Au contraire, les CE-hypotactiques sont compatibles avec les quantifieurs quasi-universels (21a,b), non-universels et les quantifieurs de masse (21c).

(19) a. Tous les élèves, sauf / excepté / (mis) à part / à l'exception de / hormis Paul, ont été punis.

b. Le maître n'a donné de livre à aucun élève, sauf à Paul.

a. *La plupart des élèves, sauf Paul, ont été punis

b. $\quad$ *Des / quelques / plusieurs / beaucoup de /peu d'élèves ont été punis, sauf Paul.

(Keiber, 2005, pp, 40-46)

(21) a. C'est dans ce cadre que doit s'illustrer l'effort consenti par la plupart des pays du monde - à l'exception de l'Inde [...]. (lemonde.fr)

b. La majorité des bureaux ont fermé, à l'exception de ceux de Nantes. (nouvelobs.com)

c. Beaucoup de joueurs, à l'exception de Nikola Karabatic, Isem Tej et Dragan Gajic, ont reconnu les paris, mais ils ont tous nié avoir triché. (lemonde.fr)

L'observation des données authentiques du français montre que les quantifieurs universels ne sont pas les seuls possibles dans les CE-paradigmatiques. Des quantifieurs tels que la plupart (22a), beaucoup de (22b), peu de (22c) sont également possibles. La 
contrainte de quantification universelle n'est pas un critère assez solide pour différencier les deux constructions.

(22) a. A la vingtaine de spectacles inscrits dans la programmation officielle, dont la plupart sont gratuits (sauf deux payants), s'ajoutent 600 compagnies accueillies dans une sorte de « off », chacune ayant son emplacement. (lemonde.fr)

b. Prénom rare, Ikram ne possède pas beaucoup de variantes, excepté Ikrame. (prenoms.com)

c. Lacoste est une marque forte, enracinée, qui contrôle sa production de $\mathrm{A}$ à $\mathrm{Z}$, ce que très peu de marques françaises font, hormis Petit Bateau. (lemonde.fr)

\subsection{Les emplois de sauf, excepté, hormis, (mis) à part en dehors de l'exception}

Il faut bien noter ici que les items sauf, excepté, hormis, (mis) à part ont d'autres emplois dans d'autres contextes où ils n'ont plus valeur d'exception et où il n'y a plus d'antécédent dans la construction. Dans ces emplois, il s'agit, comme le dit Ruozzi (2008, p.2605), de constructions qui se rapprochent des anciens usages absolus d'excepté, de sauf et d'hormis, c'est à dire d'usages dans lesquels ces participes et adjectif jouaient le rôle de prédicat et leur argument se comportait encore comme sujet de la prédication (23).

Excepté ses revenus exorbitants, son quotidien n'a pas changé. (Le Monde, cité par Ruozzi, 2008, p.2608)

Le segment introduit par excepté n'établit pas de relation de type tout-partie avec un éventuel antécédent. On ne peut plus parler d'exception. On parle dans ces cas plutôt d'une réserve $^{8}$. Le sens des items excepté, hormis, (mis) à part pourrait être glosé par 'sans compter/si l'on ne comptait pas, mettant à part/si l'on mettait à part... ${ }^{9}$ (Biardzka, 2011, p.37).

Les items excepté, hormis et (mis) à part, dans un tel emploi, se rapprochent des CEhypotactiques. Le syntagme peut se placer en tête de l'énoncé (23 \& 24) et les items excepté et (mis) à part permet aussi de placer à la fin (25).

(24) a. Hormis un incident dans le nord du pays, le premier tour des élections législatives s'est déroulé dans le calme. (lemonde.fr)

b. Mis à part la victoire de Koueires, près d'Alep, l'armée syrienne ne parvient toujours pas à avancer face aux rebelles. (lemonde.fr)

(25) a. Elle a en effet rejeté une proposition de l'Afrique du Sud qui aurait autorisé le commerce de certains sous-produits de l'éléphant, ivoire excepté. (Corpus français de Leipzig)

b. François Fillon et Benoît Hamon, candidats des deux partis de gouvernement historiques, ont des types de soutiens très similaires - les maires mis à part. (lemonde.fr)

Sauf qui exprime la réserve, quant à lui, il apparaît sous deux formes particulières : sauf$\mathrm{N}$ nu et sauf si et qui exprime la concession apparaît dans les expressions sauf à et sauf le respect. Dans le tour sauf-N nu, sauf est toujours suivi d'un nom non déterminé et parfois au pluriel. Sauf, dans cette construction, commute avec 'à moins de, sous la réserve de' ou 'sans exclure la possibilité/l'éventualité de'. Ce tour affiche les mêmes propriétés distributionnelles que les CE-hypotactiques : le syntagme introduit par sauf suivi d'un nom sans déterminant peut être antéposé (26a), inséré dans le SV (26b) ou postposé (26c).

(26) a. Sauf erreur de ma part, nos deux pays sont actuellement et seulement sur un plan juridique toujours belligérant. (lemonde.fr)

b. [...] L'expérience montre qu'en matière d'enseignement, ils n'ont, sauf exception, jamais cessé de se tromper. (lefigaro.fr)

c. Cette mesure, résultat d'un décret du 30 juillet 2008, s'appliquera automatiquement, sauf disposition contraire prise par chaque municipalité. (lemonde.fr) 
Ce tour permet aussi la coordination avec l'itération de sauf devant chaque complément :

Sauf catastrophe et sauf phénomène surnaturel, Gelabale signera ce mardi 10 octobre à Chalon. (creusot-infos.com, à propos du championnat de basket)

Sauf (et moins fréquent excepté, hormis et (mis) à part) suivis de la conjonction de subordination si expriment la réserve hypothétique (28). Leur valeur est celle de 'à moins que'.

(28) a. Sauf si on nous a sciemment menti, les comptes sont sincères. (lemonde.fr)

b. Autrement dit : l'œuvre ne survivra pas à la mort du chorégraphe, sauf si celui-ci change d'avis. (franceculture.fr)

La combinaison sauf à suivi d'un verbe à l'infinitif signifie 'sans que soit exclu le risque de' ou 'en se réservant le droit de, sans s'interdire de' (TLFi) (29a,b) qui exprime une concession. Cette locution est parfois utilisée dans le sens de 'à moins que' ou de 'sauf si' ${ }^{10}$. Enfin, l'expression sauf le respect que je lui/vous dois signifie 'sans qu'il soit porté atteinte' (TLFi) (29c,d). Dans tous ces emplois, sauf permet la position initiale ou l'insertion dans le SV et présent la possibilité de se coordonner, mais il ne s'agit en effet pas d'une exception, mais plutôt d'une réserve/concession.

(29) a. Cela ne justifie en rien d'envisager d'abandonner le recours à la justice telle qu'elle existe en France, sauf à subir une régression inconcevable. (lepoint.fr)

b. Sauf à décider d'autoriser désormais ces produits jusque-là illégaux dans l'Union européenne juste pour pouvoir les taxer, cette piste est nulle et non avenue. (lemonde.fr)

c. Sauf le respect que nous lui devons, nous espérions ne plus le revoir. (nouvelobs.com)

d. Sauf le respect dû au président américain et à ses conseillers, le problème est mal posé. (lemonde.fr)

\subsection{Récapitulatif}

Le tableau qui suit récapitule les différentes possibilités de placement linéaire du SE dans la construction, les catégories syntaxiques possibles de l'EE et la possibilité ou non de coordonner le SE.

Tableau 2. Récapitulatif les CE-paradigmatiques vs les CE-hypotactiques

\begin{tabular}{|c|c|c|c|}
\hline Critère syntaxique & & $\begin{array}{c}C E- \\
\text { paradigmatiques } \\
\text { (sauf) }\end{array}$ & $\begin{array}{c}C E- \\
\text { hypotactiques } \\
\text { (à l'exception de) }\end{array}$ \\
\hline \multirow{4}{*}{ Place linéaire du SE } & antéposition & $*$ & $\sqrt{ }$ \\
\hline & adjonction à l'Ant & $\sqrt{ }$ & $\sqrt{ }$ \\
\hline & parenthétisation & $*$ & $\sqrt{ }$ \\
\hline & postposition & $\sqrt{ }$ & $\sqrt{ }$ \\
\hline \multirow[t]{7}{*}{ Catégorie syntaxique de l'EE } & $+S N$ & $\sqrt{ }$ & $\sqrt{ }$ \\
\hline & $+S V_{i n f}$ & $\sqrt{ }$ & $*$ \\
\hline & $+S V_{\text {prép }+ \text { inf }}$ & $\sqrt{ }$ & $*$ \\
\hline & $+P_{\text {ce que }}$ & $\sqrt{ }$ & $\sqrt{ }$ \\
\hline & $+S A d v$ & $\sqrt{ }$ & $*$ \\
\hline & $+S A d j$ & $\sqrt{ }$ & $*$ \\
\hline & $+S P$ & $\sqrt{ }$ & $*$ \\
\hline $\begin{array}{l}\text { Possibilité ou non de coordonner } \\
\text { le SE }\end{array}$ & & $*$ & $\sqrt{ }$ \\
\hline
\end{tabular}

La coordination se distingue de la subordination par un certain nombre de critères (cf. Tesnière, 1959 ; Mouret, 2007) parmi lesquels (i) la compatibilité syntaxique des éléments conjoints, c'est-à-dire la conjonction d'éléments de même nature et fonction, (ii) 
l'impossibilité d'antéposer ou de coordonner la séquence que les conjonctions de coordination introduisent. Les propriétés distributionnelles et combinatoires du SE supra nous permettent finalement de conclure que les CE-paradigmatiques s'apparentent, du point de vue syntaxique, à la coordination et que les CE-hypotactiques relèvent au contraire de la subordination. Nous lançons, par conséquent, l'hypothèse que les ME dans l'emploi paradigmatique sont des conjonctions de coordination, alors que les ME dans l'emploi hypotactique peuvent être analysées comme des prépositions. Dans la suite, nous présenterons notre analyse des CE-paradigmatiques comme un cas particulier de listes paradigmatiques et celle du marqueur de ces constructions comme une conjonction de coordination.

\section{Les constructions exceptives en sauf, excepté, hormis, etc. comme un cas de listes paradigmatiques}

Nous proposons de traiter les ME sauf, excepté, hormis, etc. comme des conjonctions de coordination qui mettent en relation deux éléments $\mathrm{X}$ et $\mathrm{Y}$, l'un à droite, l'autre à gauche du $\mathrm{ME}$, le tout formant une liste paradigmatique occupant une unique position syntaxique dans l'énoncé (cf. Blanche-Benveniste, 1990 ; Gerdes \& Kahane, 2009 ; 2015 ; Guénot, 2005 ; Kahane et Pietrandrea, 2012 ; Nølke, 1983). Par exemple, si on reprend l'exemple (6a), où $\mathrm{X}=$ tous les partis et $\mathrm{Y}=l^{\prime} U M P$, on constate que $[\mathrm{X}$ sauf $\mathrm{Y}]$ forme un syntagme qui remplit le rôle de sujet du verbe veulent. De plus, X et Y sont bien dans le même paradigme et chacun d'eux pourrait occuper à lui seul la position qu'occupe [X sauf $\mathrm{Y}]$.

(6) a. Tous les partis, sauf l'UMP, veulent une "dose" de proportionnelle aux législatives. (lemonde.fr)

Plusieurs phénomènes en français entrent dans la définition des entassements paradigmatiques, comme la coordination, la reformulation ou même certaines séquences question-réponse (dont nous verrons des exemples plus loin). Jusqu'ici les CE n'avaient pas été considérées (voir la classification proposée par Kahane et Pietrandrea, 2012).

L'exception peut être analysée de la même manière que la coordination (30). Nous proposons pour l'exemple (31a) une représentation en syntaxe de dépendance en $(31 \mathrm{~b})$ où sauf prend la catégorie $\mathrm{COO}$ (conjonctions de coordination), tiré par la relation mark (la relation entre la tête d'un terme de la liste paradigmatique et la conjonction de coordination) et la relation para entre l'Ant et l'EE (cette relation représente un lien paradigmatique qui rattache un élément à son conjoint le plus proche au sein de la liste. Le premier conjoint de la liste est la tête et le gouverneur de la liste s'y rattache $)^{11}$. L'annotation des exemples suit les conventions du corpus Orféo (cf. Kahane et al., 2016 ; 2017).

(30) a. Nous avons [trois filles] et [un garçon].

b.

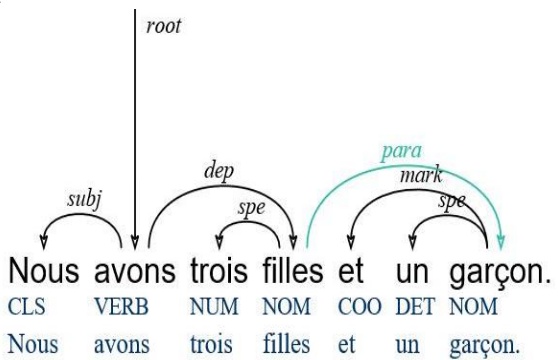

(31) a. je travaille [tous les jours], sauf [le lundi].

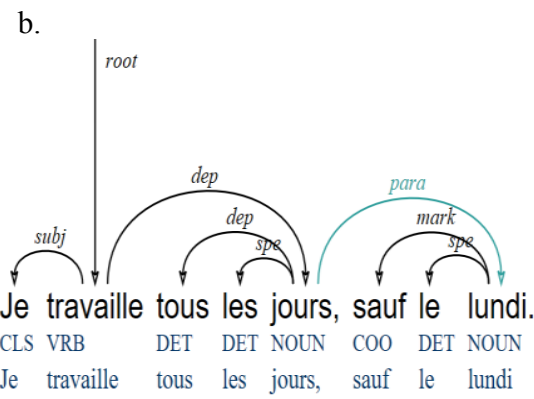



(32).

L'analyse sera la même quelle que soit la nature du syntagme introduit : un SP dans

(32) a. Alexandre Tharaud joue du piano [partout], sauf [chez lui]. (lemonde.fr)

b.

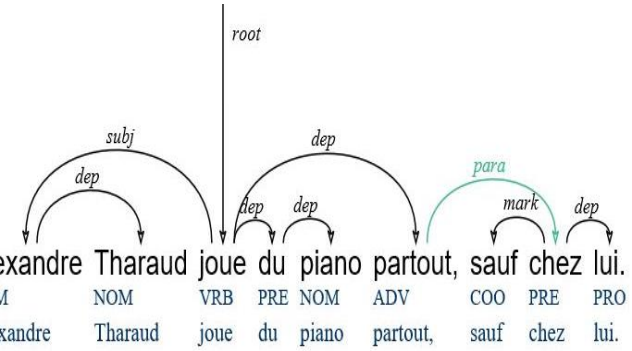

\subsection{Les spécificités de la construction exceptive paradigmatique par rapport à la coordination}

Il existe un certain nombre de propriétés qui distinguent, ou semblent distinguer, les CEparadigmatiques par rapport à la coordination et que nous présentons dans la suite.

\subsubsection{Une asymétrie forte}

Contrairement aux autres entassements paradigmatiques, les CE présentent une asymétrie inconditionnelle entre les conjoints, puisque ceux-ci ne peuvent pas être permutés :

(33) a. Je travaille tous les jours, sauf le lundi.

b. *Je travaille le lundi, sauf tous les jours.

Il existe des constructions paradigmatiques comme la coordination en mais qui sont également asymétriques, mais la permutation reste possible: elle crée un énoncé sémantiquement différent, mais, néanmoins grammatical :

(34) a. L'EBA ne s'installera pas à Luxembourg, mais à Paris. (wort.lu, à propos du Brexit)

b. L'EBA ne s'installera pas à Paris, mais à Luxembourg.

\subsubsection{L'absence d'antécédent}

Les CE-paradigmatiques peuvent apparaître sans la présence explicite de l'Ant du type $[\{\mathrm{X}\}$ sauf $\mathrm{Y}]$, notamment dans le cas où l'EE fonctionne comme un circonstant du prédicat de la proposition principale, comme dans (35). La raison pour laquelle l'Ant n'est pas explicite dans l'énoncé principal, revient, comme l'a noté Ruozzi (2008, p.2605), au fait qu'il s'agit de rôle conceptuel extranucléaire et que, par conséquent, son codage n'est pas obligatoire, mais facultatif, le prédicat dont l'Ant dépend peut aussi choisir de le coder ou non.

(35) a. Le parking est public, sauf les matinées du marché Grandclément. (leprogres.fr)

b. Le parking est \{toujours\} public, sauf [les matinées du marché Grandclément].

En fait, cette propriété ne distingue pas les CE-paradigmatiques des coordinations. L'absence d'un premier conjoint se produit également avec la coordination. Les coordinations en et où mais aussi peuvent ne pas avoir d'antécédent (Gerdes \& Kahane, 2009) :

(36) a. Il parle le français et bien. 
b. Il parle le français, mais mal.

Dans les exemples en (36), le syntagme introduit par la conjonction de coordination (bien ; $\mathrm{mal}$ ) ne s'inscrit pas le même paradigme qu'un éventuel premier conjoint. Il s'agit, en effet, comme le dit Gerdes \& Kahane (2015, p.109), d'une coordination entre deux unités illocutoires : (36a) véhicule ainsi deux assertions, 'il parle le français' et 'il le parle bien'. Voilà un exemple en mais sans antécédent tiré de corpus Orféo :

a. Il frappe de loin mais [dans les nuages].

b.

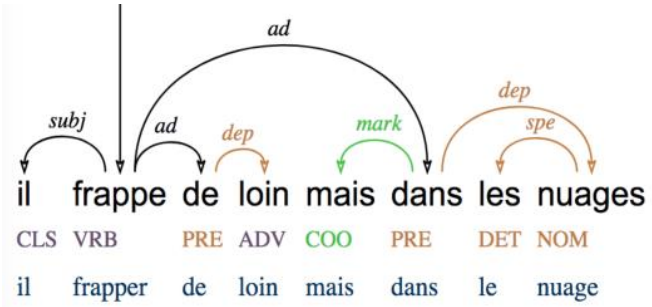

Dans tels cas, il n'y a pas de lien relation paradigmatique (cf. la relation para dans les figures $30 \mathrm{~b}$ et $31 \mathrm{~b}$ ), mais on a toujours la relation mark pour le marqueur (38).

(38) a. Le parking est public, sauf [les matinées du marché Grandclément].

b.

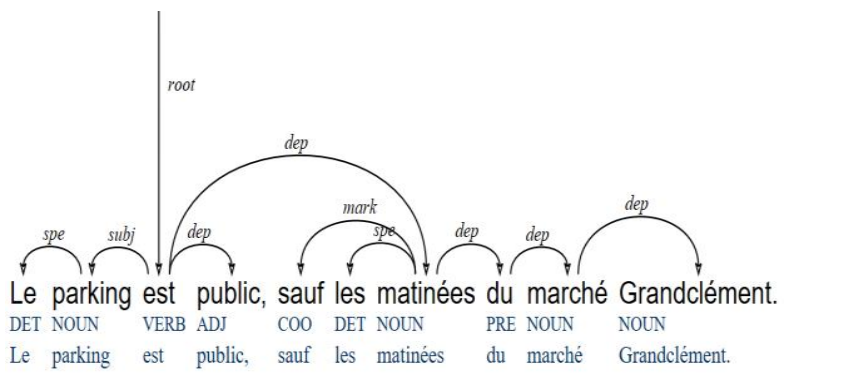

\subsubsection{La discontinuité}

Comme nous l'avons déjà montré plus haut, l'impossibilité pour le SE d'être antéposé dans les CE-paradigmatiques justifie le rapprochement avec la coordination :

(39) a. Pratiques et discours diffèrent considérablement aux États-Unis, terre de common law, et en France. (odilejacob.fr)

b. *Et en France, pratiques et discours diffèrent considérablement aux États-Unis, terre de common law.

Par contre, le fait que le SE peut être postposé et n'être plus adjacent à l'Ant peut apparaître comme une différence avec la coordination, puisqu'on considère généralement que les conjoints d'une coordination doivent être contigus. Pourtant, il est possible pour un deuxième conjoint d'être placé en fin d'énoncé, sans être adjacent au premier conjoint. Il s'agit d'un cas particulier de complément différé ${ }^{12}$ :

(40) a. Le nerf, c'est "l'affaire Grégory" qui me l'a donnée. Ou plutôt, moins "l'affaire" que son contexte. (Est Républicain)

b. Cela vient de l'école, ici, on est puni si on coupe la parole à un camarade. Et d'une tradition rurale encore très forte. (Est Républicain)

c. A la mi-temps, alors que le score est toujours vierge, le public est loin d'être faraud. Et Alain Jankowski pas plus que les autres. (Est Républicain) 
d. Nous avons choisi aussi de prendre part à la mondialisation d'en prendre toute notre part / mais une mondialisation maîtrisée organisée (oral, Rhapsodie)

La postposition du SE n'exclut donc pas de traiter les CE-paradigmatiques comme des listes/entassements paradigmatiques. Gerdes \& Kahane (2009) et Kahane \& Pietrandrea (2012) classent d'ailleurs parmi les entassements paradigmatiques des constructions comme l'effet deux points (41a) ou les réponses partielles (41b), où le deuxième conjoint est systématiquement postposé.

(41) a. Je dirais que vous avez donné quelque chose de plus à la femme : des armes de persuasions. (oral, Rhapsodie-corpus Mertens, nous rajoutons la ponctuation)

b. euh pourquoi j'ai fait du journalisme ? parce que euh ça se passait tout de suite après la guerre. (oral, Rhapsodie-corpus Mertens, nous rajoutons la ponctuation)

Nous donnons en (42) un exemple d'analyse d'une coordination discontinue extraite du corpus Orféo et en (43) une analyse d'un CE-paradigmatique discontinue analysée selon le même schéma d'annotation.

(42) a. [...] de prendre part à la [mondialisation] d'en prendre toutes notre part mais [une mondialisation maîtrisée] [...] (Corpus Orféo)

b.

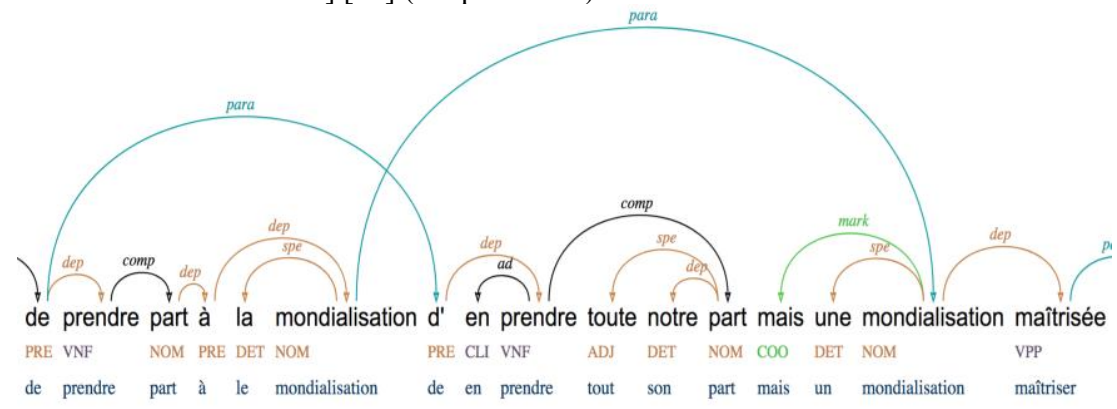

(43) a. [Toutes les couleurs] de robes sont admises sauf [les couleurs chocolat et lilas]. (Corpus français de Leipzig)

b.

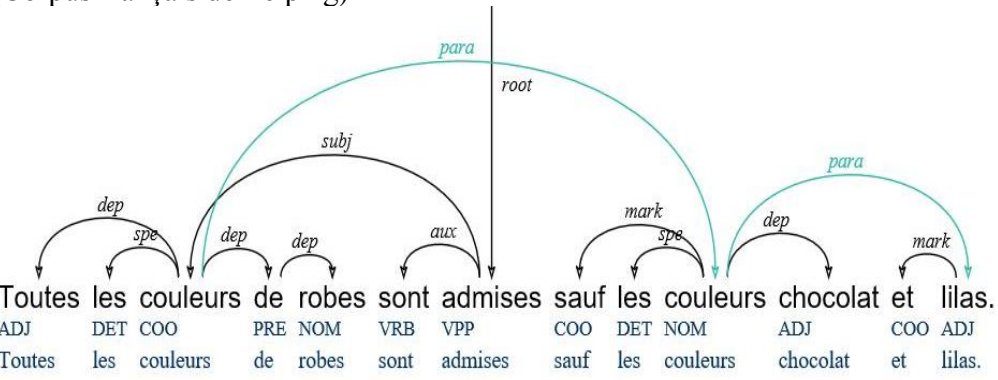

Pour les ME des CE-hypotactiques en tant que locutions prépositives, nous proposons une représentation syntaxique de l'énoncé (44a) en (44b). La relation periph est celle qui relie les éléments périphériques, en position détachée, à la tête de l'énoncé (cf. Benzitoun et al., 2010 ; Gerdes \& Kahane, 2015).

(44) a. À l'exception de la CGT, tous les syndicats affichent une positive attitude. (lemonde.fr) 
b.

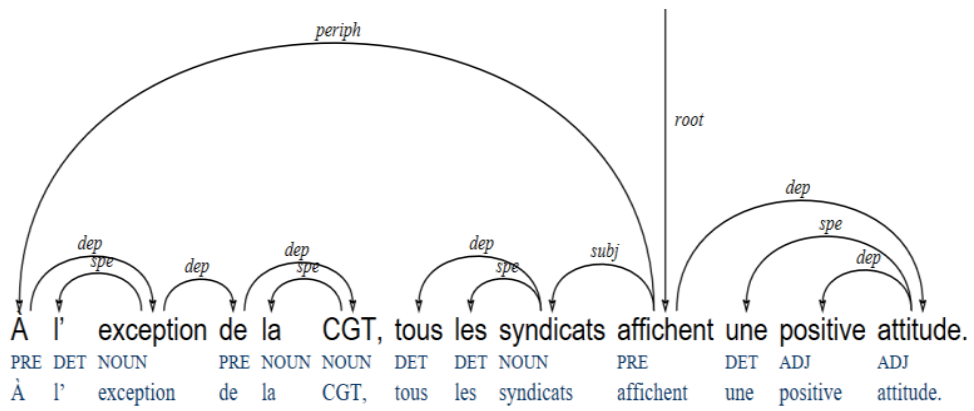

\section{La catégorie syntaxique des marqueurs exceptifs}

La question de la catégorie syntaxique des $\mathrm{ME}$ a entraîné nombre de polémiques dans la littérature existante sur les CE dans de nombreuses langues, français compris. Dans cette section, nous allons donner des arguments supplémentaires en faveur du traitement des marqueurs des CE-paradigmatiques comme des conjonctions de coordination, après avoir discuté les propositions de les classer comme prépositions, adverbes ou conjonctions de subordination.

\subsection{Le classement comme préposition}

Les marqueurs sauf, excepté, hormis, etc. en français ont été communément analysés comme des prépositions dans les grammaires et les dictionnaires consultés (cf. Grevisse et Goosse 2008; Le Goffic, 1993 entre autres). C'est également cette analyse qui est actuellement adoptée par les corpus du français disponibles dans les treebanks Universal Dependencies $^{13}$ (UD). En (45), sauf, excepté, hormis prennent la catégorie ADP (adposition).

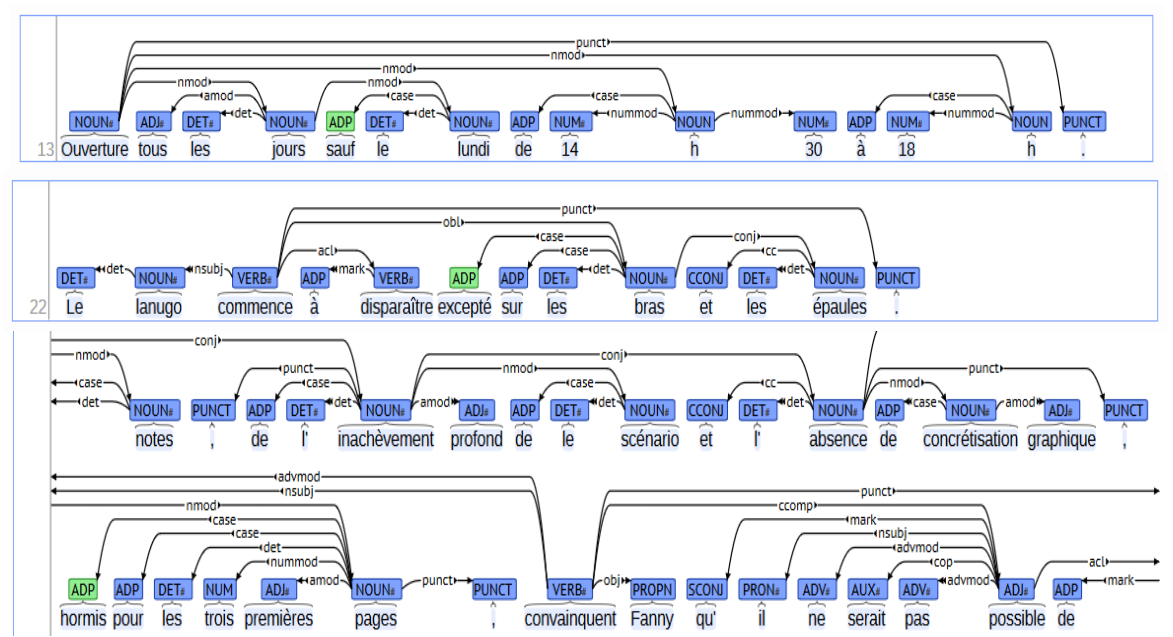

Creissels (1995, pp.184-195) a proposé un argument contre le traitement de sauf comme préposition. Il remarque que l'une des propriétés principales des compléments prépositionnels du verbe est de pouvoir se trouver sous la portée d'une négation. Dans l'exemple en (46a), la négation affecte sémantiquement le complément prépositionnel et non pas le verbe lui-même. Une interprétation possible de (46a) est que Michel est venu par 
un autre moyen que sa voiture. La phrase (46b) exprime la même signification sans aucune ambiguïté.

(46) a. Michel n'est pas venu avec sa voiture.

b. Michel est venu, mais pas avec sa voiture.

(Creissels, 1995, p.184)

Or la présence de sauf dans une phrase va de pair avec un blocage de la portée de la négation. L'exemple en (47a) n'admet pas l'introduction d'une négation : les exemples en (47b-c) ne constituent pas des phrases acceptables. Inversement, (48a) est normalement la forme négative de (48b), et pourtant (48c) ne saurait être considérée comme la forme négative de (48d), et (48e) n'est pas une paraphrase de (48c), puisque (48d) et (48e) sont aussi inacceptable que (47b) et (47c).

(47) a. Je les ai tous vus sauf Michel.

b. *Je ne les ai pas tous vus sauf Michel.

c. *Je les ai tous vus, mais pas sauf Michel.

(48) a. Je n'ai vu personne.

b. J'ai vu quelqu'un.

c. Je n'ai vu personne sauf Michel.

d. *J'ai vu quelqu'un sauf Michel.

e. *J'ai vu quelqu'un, mais pas sauf Michel.

(Creissels, 1995, p.185)

Le test nous semble difficile à appliquer aux $\mathrm{CE}$ en raison des interférences complexes entre la négation et le quantifieur universel. Mais surtout, (47b) reste inacceptable si on remplace sauf par à l'exception de. Le test proposé par Creissels ne discrimine donc pas entre les CE-paradigmatiques et les CE-hypotactiques et ne permet pas de conclure que sauf est n'est pas une préposition, pas plus qu'il ne permet de décider si à l'exception de est une préposition ou pas.

Les arguments essentiels contre le classement comme préposition sont, au final, ceux qu'on a déjà donnés à la section 2 : la possibilité pour les ME dans l'emploi paradigmatique de se combiner avec des syntagmes de différentes parties du discours et les contraintes sur la place linéaire qui sont différentes de celles des groupes prépositionnels ${ }^{14}$.

\subsection{Le classement comme adverbe}

Les ME sauf, excepté, hormis, etc. ont des analogies avec les adverbes paradigmatiques de focalisation, tels que seul, aussi, même, notamment, etc. :

(49) a. Tout le monde est là, sauf Pierre.

b. Tout le monde est là, même Pierre

Ces analogies sont à la fois syntaxique (position similaire) et sémantique : ils marquent le rôle d'un élément parmi le paradigme des éléments qui peuvent commuter avec lui. Ils ont notamment les mêmes contraintes sur le rapport d'inclusion entre les deux éléments introduits :

(50) a. *Tous les étudiants sont venus, sauf leur professeur.

b. *Tous les étudiants sont venus, même leur professeur.

Par contre, même ne nécessite pas la mise en relation de deux conjoints :

(51) a. Paul a invité tout le monde, même Pierre.

b. Paul a invité tout le monde, sauf Pierre.

c. Paul a invité même Pierre.

Cette dernière propriété distingue les adverbes paradigmatiques des conjonctions de coordination. La deuxième propriété qui les distingue est que, comme nous l'avons déjà signalé plus haut, les ME sauf, excepté, hormis ne sont pas compatibles avec les 
conjonctions de coordination, alors que les adverbes paradigmatisants peuvent être précédés par une conjonction de coordination (52):
Pour les accueillir, des startups proposent la location temporaire de bureaux ou de salles de conférence partout dans le monde. ... Canada, en Allemagne, en Espagne, en Grande- Bretagne, en Australie, à Hong Kong, à Singapour, aux Pays-Bas, au Mexique, en Corée du Sud, en Inde et même en France. (lesechos.fr)

\subsection{Le classement comme conjonction de subordination}

La troisième étiquette a été proposée par Piot (2005), considérant que les ME introduisant une séquence nominale ou infinitivale en français, soit en forme simple sauf, excepté, etc. ou en forme conjonctive sauf que, excepté que etc. en parallélisme aux comparatives sont de pures conjonctions de subordination. L'argumentation se base sur le parallélisme avec les constructions comparatives en comme, comme illustré dans les exemples en (53).

(53) a. J'avais tout prévu, sauf (ton départ/de m'en aller).

b. J'avais tout prévu, sauf [que je n'avais pas prévu] (ton départ/de m'en aller).

c. Pierre ne boit pas de whisky, comme de vins trop corsés.

d. Pierre ne boit pas de whisky, comme [il ne boit pas] de vins trop corsés.

(Piot, 2005, pp.297 et 301)

Bien que le parallélisme entre sauf et comme est valable, nous considérons cependant que cette étiquette n'est pas non plus pertinente. Comme l'ont noté Kahane \& Pietrandrea (2012), les constructions comparatives se voient aussi comme des entassements paradigmatiques. Voir par exemple l'analyse de plus que dans le treebank Orféo :

(54) a. Votre marché c'est Aligre plus que Nation. (Rhap-D0001, CFPP2000)

b.

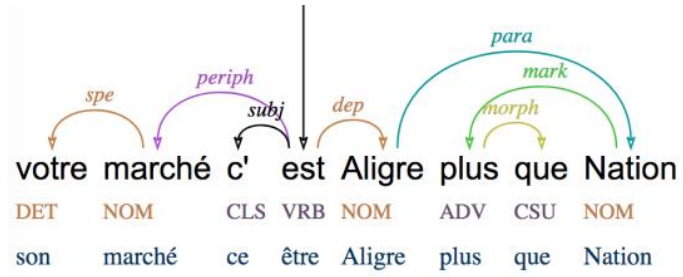

De plus, on ne peut pas non plus cliver les constructions en comme, alors que les conjonctions de subordinations permettent d'être clivés :

(55) a. *C'est comme de vins corsés que Pierre ne boit pas de whisky.

b. C'est parce qu'il pleut qu'il ne vient pas.

Même dans ses autres emplois l'analyse de comme comme une conjonction de subordination pose de problèmes puisque cet élément est compatible avec les conjonctions de subordination :

(56) a. Le sol est mouillé comme quand il pleut.

b. Le sol est mouillé comme s'il avait plu.

\subsection{Le classement comme conjonction de coordination}

La quatrième étiquette associée aux ME est celle de conjonction de coordination proposée, pour l'anglais d'abord, par Harris (1982) et Reinhart (1991), mais aussi plus récemment par García Álvarez (2008), puis pour l'espagnol par Pérez-Jimenéz et Mareno-Quibén (2012) et pour l'arabe égyptien par Soltan (2016). 
Reinhart (1991) a proposé une analyse par la représentation en forme logique (angl. Logical Form (LF)) où le SE s'adjoint sur l'expression quantifiée. La structure de surface est donnée en (57a), la LF en (57b) et la structure profonde en (57c).

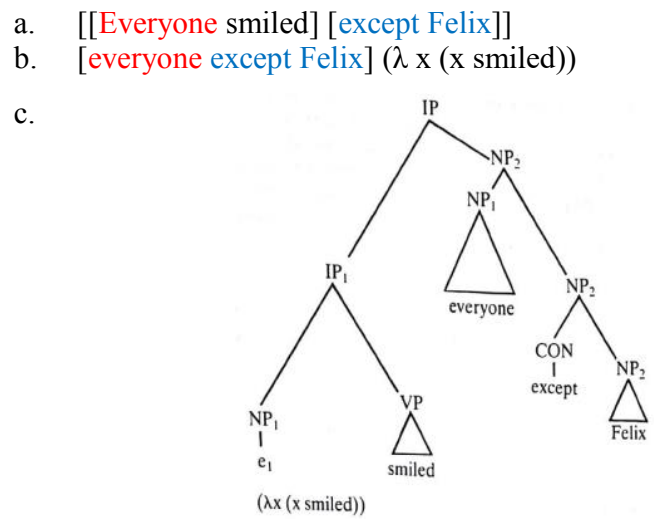

(Reinhart, 1991, p.367)

Cette proposition, comme l'a noté Hoeksema (1995, pp.47-48), s'approche de celle suggérée originairement par Harris (1982) qui a traité, lui aussi, except/but comme des conjonctions de coordination. Harris a considéré que les ME coordonnent non pas, comme dans l'analyse de Reinhart, deux SN, mais deux propositions. Selon lui, une série de réductions (zeroing transformations) a lieu dans la deuxième proposition (58) :

(58) a. Everyone smiled, except Felix did not smile.

b. Everyone smiled, except Felix did not.

c. Everyone smiled, except not Felix.

d. Everyone smiled, except Felix.

(Hoeksema, 1995, pp.147-148)

En 1987, Hoeksema a introduit une typologie binaire des CE basée sur les propriétés distributionnelles du SE et sur les possibilités combinatoires de la construction. Il a nommé la première construction "connected exceptive " et la deuxième "free exceptive ", ce que nous traduisons désormais par exceptive connectée et exceptive libre.

Les deux types sont canoniquement illustrés en anglais en (59) :
Connectée
a. Every day but/except Sunday it was raining.
Libre
b. Except for Sunday, it was raining every day.
(Hoeksema, 1987, p.100)

D’une façon générale, le SE except Sunday, dans les exceptives connectées, est associée à un SN contenant obligatoirement un quantifieur universel every dont le SE sert à limiter la portée universelle. Au niveau de la place syntaxique, le SE est soit adjacent au SN qu'il modifie (59a), soit postposé dans la construction (60a). Une position initiale dans l'énoncé n'est pas possible (60b). En revanche, le SE, dans les exceptives libres, occupe une position adverbiale et a une distribution plus libre : il peut s'antéposer (59b), se parenthétiser (60c) ou se postposer (60d). Les exceptives libres de l'anglais, contrairement aux exceptives connectées, sont compatibles avec les quantifieurs non-universels most, few, etc., quasiuniversels the majority, aussi bien qu'avec les phrases génériques.

(60) a. It was raining every day but/except Sunday.

b. *But/except Sunday, it was raining every day.

c. It was, except for Sunday, raining every day.

d. It was raining every day, except for Sunday.

(Hoeksema, 1987, p.100)

Hoeksema (1987, pp.101-102) et von Fintel (1993, pp.17-18), à la suite de Horn et Bayer (1984), ont postulé aussi que les exceptives libres sont compatibles avec les interrogatifs (61a), alors que les exceptives connectées ne le sont pas (61b). Les exceptives 
connectées sont seulement compatibles avec les questions rhétoriques, comme (61c) où who est commutable avec nobody.

(61) a. Except for John, who is coming to the party?

b. ?*Who but Leslie is coming to a party?

c. Who but a total idiot would have said a thing like that?

(Hoeksema, 1987, p.102)

(von Fintel, 1993, p.18)

(von Fintel, 1993, p.18)

En se basant sur les différences portant sur la position syntaxique et les possibilités combinatoires entre les exceptives connectées et les exceptives libres, Hoeksema (1995) a proposé de considérer les exceptives connectées comme des modifieurs adnominaux (postmodifiers of noun phrase) et les exceptives libres comme des modifieurs de phrase (sentence modifier). Hoeksema a noté aussi, à la suite de Harris (1982), que l'on peut analyser les $\mathrm{CE}$ comme un cas de constructions trouées ${ }^{15}$ de la coordination où la séquence qui suit le ME est le reste d'une construction elliptique. Il a également noté que les ME dans les exceptives libres ont un comportement qui les rapproche des prépositions et non des conjonctions de coordination.

Nous partageons avec Hoeksema $(1987$; 1995) la description qu'il a proposée des propriétés distributionnelles des deux types de constructions en cause. Ces propriétés sont d'ailleurs confirmées en français comme nous l'avons déjà vu dans notre classification des CE. Nous ne retiendrons néanmoins pas les autres hypothèses associées à cette taxonomie. Premièrement, comme nous l'avons déjà dit dans la section 2.4, le quantifieur universel de l'Ant n'est pas le seul possible dans les exceptives connectée en sauf, excepté, hormis en français. Soulignons que l'hypothèse de la quantification universelle a été rejetée aussi par García Álvarez (2008) qui justifie, en se basant sur des données attestées de l'anglais, que les constructions exceptives connectées sont aussi compatibles avec des expressions de quantification non-universelle du type most, many et few :

(62) a. Kate is an actress who has played many roles except that of a real woman.

b. Karadzic is a moderate man in most things but politics.

(García Álvarez, 2008, p.13, 114)

Deuxièmement, bien qu'étant d'accord avec Hoeksema (1987) et von Fintel (1993) sur le fait que les CE-paradigmatiques en français ne sont pas compatibles avec les questions informatives (63a) et qu'elles acceptent seulement les questions rhétoriques (63b), nous ne pensons pas que les CE-hypotactiques soient compatibles avec les questions informatives. Comme l'a noté Hoeksema (1987, p.101) lui-même, à la suite de Landman et Moerdijk (1980), dans ces exemples où les CE sont compatibles avec les questions informatives, le sens du ME glisse vers l'additif et peut être commuté avec en plus de (63c). Dans l'emploi intrinsèquement exceptif, les CE-hypotactiques en français, comme les CEparadigmatiques, ne sont pas compatibles avec les interrogations informatives (63d).

(63) a. *Qui sauf Pierre viendra te chercher à l'aéroport ?

b. Qui sauf Mme Romanin, si elle est un peu voyante à ses heures, aurait pu se douter qu'un concert comme celui - là remplirait la cathédrale Saint-Benoit? (lemonde.fr)

c. Qui à part moi regrette le temps des acteurs couillus qui étaient de vrais hommes ? (allocine.fr)

d. *Qui à l'exception de Pierre viendra te chercher à l'aéroport ?

Plus récemment, García Álvarez (2008), Pérez-Jimenéz et Mareno-Quibén (2012) et Soltan (2016) ont repris la typologie de Hoeksema $(1987$; 1995). Selon eux, les ME dans les deux types, exceptives connectée et exceptives libres, sont des conjonctions de coordination. La différence est dans le type des syntagmes conjoints : dans les exceptives connectées, deux SN ('DP level' constituents) sont conjoints, alors que, dans les exceptives libres, deux propositions enchâssées ('sentence level' constituents) sont conjointes et une ellipse a lieu dans la deuxième proposition. 
Ces analyses des ME comme conjonctions de coordination en anglais, en espagnol et en arabe égyptien sont confirmées par nos analyses des ME en français. Nous considérons que les $\mathrm{ME}$ en français dans l'emploi paradigmatique partagent avec les conjonctions de coordination les mêmes propriétés définitoires : (i) la non-combinabilité des conjonctions entre elles (64), (ii) leur absence de mobilité dans le domaine dans lequel elles apparaissent (65) et (iii) l'impossibilité d'extraire (66), d'antéposer (67) ou de coordonner la séquence qu'elles introduisent (68).

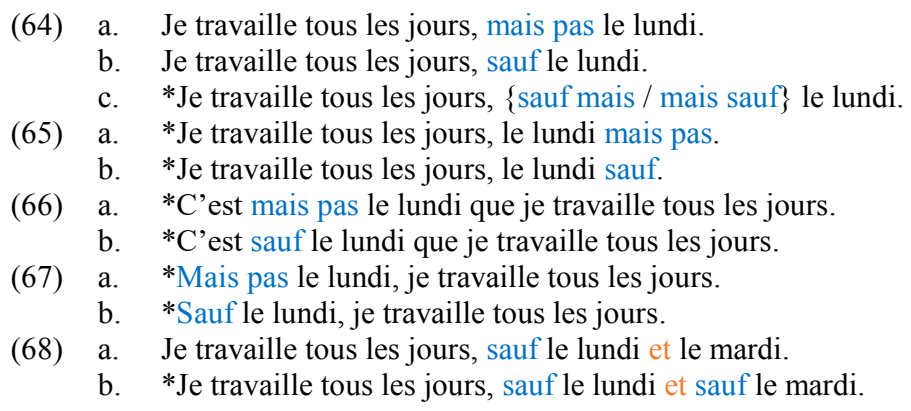

\section{Conclusion}

Nous avons proposé dans cette étude une description de la syntaxe des constructions exceptives en français contemporain. Les analyses présentées mettent en évidence qu'il existe deux types de constructions exceptives avec des comportements syntaxiques distincts.

Les $C E$-paradigmatiques établissent une relation paradigmatique entre les deux termes d'exception, l'antécédent et l'élément excepté. Dans ces constructions, la place linéaire du syntagme exceptif est soit adjacent à son antécédent, soit postposé. Les marqueurs qui introduisent ces constructions peuvent se combiner avec une grande variété de syntagmes (SN, SP, SAdv, etc.) et le syntagme exceptif ne permet pas la coordination. Ces caractéristiques montrent que les CE-paradigmatiques s'apparentent aux entassements/listes paradigmatiques dont le cas le plus connu est la coordination. Nous avons montré, à l'appui de notre hypothèse, l'analogie entre les marqueurs exceptifs dans l'emploi paradigmatique et les conjonctions de coordination.

Les CE-hypotactiques établissent une relation d'hypotaxe entre la proposition principale et le syntagme exceptif. Dans ces constructions, la distribution du syntagme exceptif est plus libre. Il accepte le détachement en tête de l'énoncé, la parenthétisation et la postposition. Les marqueurs qui introduisent ces constructions ne se combinent qu'avec un substantif et le syntagme exceptif permet la coordination. Ces propriétés montrent que les marqueurs dans cet emploi ont un comportement de prépositions.

Nous avons considéré les CE-paradigmatiques comme des cas particulier de constructions paradigmatiques, ce qui n'avait pas été remarqué précédemment, ni dans les travaux sur les constructions paradigmatiques (cf. Blanche-Benveniste, 1990 ; Kahane et Pietrandrea, 2012) ni dans les travaux sur les constructions exceptives qui avaient fait le lien avec la coordination, mais sans introduire le concept de construction paradigmatique et proposer une analyse syntaxique unificatrice de ces phénomènes.

\section{Références bibliographiques}

Benzitoun C., et al. (2010). tu veux couper là faut dire pourquoi : propositions pour une segmentation syntaxique du français parlé, CMLF, La Nouvelle Orléans, 139-155. 
Biardzka, E. (2011). Exception, restriction, mis à part sur le sens hétérogène des constructions prépositionnelles dites « exceptives », Romanica Cracoviensia, 11, 32-40.

Blanche-Benveniste, C. et al. (1990). Le français parlé : études grammaticales, Paris : CNRS.

Botalla M.-A. (à paraître). Modélisation de la production des énoncés averbaux : le cas des compléments différés. Thèse de l'Université Paris 3 Sorbonne Nouvelle.

Choi-Jonin, I. et Migno, F. (2011/2). Les emplois de sinon et le processus d'interprétation, Travaux de linguistique, 63, 59-84.

Creissels, D. (1995). Éléments de syntaxe générale. Paris : Presses Universitaires de France-PUF.

Gajewski, J. (2008). Npi any and connected exceptive phrases, Language Semantics, 16(1). 69-110.

García Álvarez, I. (2008). Generality and exception. A study in the semantics of exceptives. Thèse de Doctorat, SU.

Gerdes K. et Kahane S. (2009). Speaking in piles: Paradigmatic annotation of French spoken corpus, Proceedings of the Fifth Corpus Linguistics Conference, Liverpool.

Gerdes K. et Kahane S. (2015). Non-constituent coordination and other coordinative constructions as dependency graphs, Depling, Uppsala.

Grevisse, M. et Goosse, A. (2008). Le bon usage. Grammaire française. Bruxelles: De BoeckDuculot.

Guénot, M-L. (2005). La coordination considérée comme un entassement paradigmatique : description formalisation et intégration, CENTAL, 178-187.

Harris, Z. (1976). Notes du cours de syntaxe. Pais : Du Seuil.

Harris, Z. (1982). A Grammar of English on Mathematical Principles; New York: John Wiley \& Sons.

Hoeksema, J. (1987). The logic of exception, in: Miller, A., Powers, J., Proceedings of the Fourth Eastern States Conference on Linguistics, Columbus, OH, 100-113.

Hoeksema, J. (1995). The semantics of exception phrases, Quantifiers, logic, and language, 145 -177.

Ionescu, E. (2013). Exceptive Phrases in Romanian. A Fragment-based Analysis, The 20th International Conference on Head-Driven Phrase Structure Grammar.

Kahane S. et al. (2016). Guide d'annotation syntaxique Orféo (version Platinum).

Kahane S. et Pietrandrea P. (2012). La typologie des entassements en français, CMLF, Lyon, 18091828.

Kahane, S. et al. (2017). Annotation micro- et macrosyntaxique manuelle et automatique de français parlé, Journée Floral, Orléans.

Kleiber, G. (2005). Comment se "règle » linguistiquement l'exception : petite sémantique des constructions exceptives, Faits de langues, 25, 39-52.

Lappin, S. (1996). Generalized quantifiers, exception phrases, and logicality, Journal of Semantics, 13, 197-220.

Le Goffic, P. (1993). Grammaire de la phrase française. Paris : Hachette.

Léard, J.M. et Marengo, S. (2006). L'exception et la restriction : similitude, différence ou inclusion ?, in : La relation partie-tout (éd. Georges Kleiber, Catherine Schnedecker, Anne Theissen, Peeters, Leuven).

Moignet, G. (1959). Les signes de l'exception dans l'histoire du français. Genève : Droz.

Moltmann, F. (1992). Coordination and comparatives. Thèse de Doctorat, MIT.

Moltmann, F. (1995). Exception sentences and polyadic quantification. Linguistics and Philosophy, $18,223-280$. 
Mouret, F. (2007). Grammaire des constructions coordonnées. Coordinations simples et coordinations à redoublement en français contemporain. Thèse de Doctorat, Paris VII.

Mourin, L. (1980). L'exception et la restriction dans les langues romanes, Travaux de Linguistique et de Littérature, 18, 173-175.

Munn, A., (1993). Topics in the syntax and semantics of coordinate structures. These de Doctorat, University of Maryland, College Park.

Nølke, H. (1983). Les adverbes paradigmatisants : fonction et analyse. Copenhague: Akademisk Forlag.

Pérez-Jimenéz I. et Mareno-Quibén N. (2012). On the Syntax of Exceptions. Evidence from Spanish, Lingua, 122, 582-607.

Piot, M. (1995). Composition transformationnelle des phrases par subordination et coordination, Thèse de Doctorat, Paris 7.

Piot, M. (2005). Sur la nature des fausses prépositions sauf et excepté, French Language Studies, 5, 297-314.

Potsdam E. et Polinsky M. (2017). A Preliminary Look at Exceptives in Tahitian, in: Asking the Right Questions: Essays in Honor of Sandra Chung, 28-36.

Reinhart, T. (1991). Elliptic conjunctions - non-quantificational QR, in: Kasher, A. (Ed.), The Chomskian Turn. Blackwell, Cambridge, MA, 360-384,

Roig, A. et Rosier, L. (2012). «Rien de nouveau... mis à part plusieurs pensées futiles », Arts et Savoirs [En ligne], 2.

Rouzzi, P. (2008). La relation de limitation et d'exception dans le français aujourd'hui : «excepté », « sauf » et « hormis » comme pivots d'une relation algébrique, $C M L F^{\prime} 08,2601-2614$.

Soltan, U. (2016). On the syntax of exceptive constructions in Egyptian Arabic, Perspectives on Arabic linguistics, XXVII, (éd. Stuart Davis and Usama Soltan).

Tesnière L. (1959). Eléments de syntaxe structurale. Paris : Kliencksieck.

von Fintel, K. (1993). Exceptive constructions, Natural Language Semantics, 1, 360-384.

\footnotetext{
${ }^{1}$ Nous montrerons plus loin (\$2.4) que cette condition peut ne pas être complètement remplie pour le français.

${ }^{2}$ Voir Choi-Jonin et Mignon (2011) sur les emplois de sinon.

${ }^{3}$ On oppose généralement paradigmatique à syntagmatique et hypotactique à paratactique. Comme l'a souligné Blanche-Benveniste (1990), les constructions paradigmatiques sont aussi syntagmatiques, puisque les deux conjoins entretiennent à la fois une relation paradigmatique (possibilité de commuter l'un avec l'autre) et une relation syntagmatique (ils se combinent l'un avec l'autre). Par ailleurs, il ne s'agit pas d'une construction paratactique puisque la construction possède un marqueur bien identifiable (sauf, excepté, etc.)

${ }^{4}$ Cette typologie a aussi été reprise dans un certain nombre des travaux en sémantique de l'exception (Crnič, 2016 ; von Fintel, 1993 ; Gajewski, 2008 ; García Álvarez, 2008 ; Moltmann, 1992 ; 1995) aussi bien qu'en syntaxe de l'exception dans des travaux sur l'espagnol par Pérez-Jimenéz et MarenoQuibén (2012) et sur l'arabe égyptien par Soltan (2016).

${ }_{5}^{5}$ Notons ici que, d'après Choi-Jonin et Mignon (2011), sinon dans son emploi exceptif est en corrélation avec une proposition négative (i) et dans l'emploi restrictif est en corrélation avec une proposition interrogative introduit une réponse anticipée que l'on présente comme étant la seule possible (ii). Les exemples cités par Choi-Jonin \& Mignon (2011, p.59).

(i) Je ne sais plus bien ce qui me maintient encore en vie sinon l'habitude de vivre. (Gide)

(ii) À quoi cette poésie peut-elle servir, sinon à égarer notre bon sens? (Hugo).

${ }^{6}$ Les locutions conjonctives sauf que, excepté que, hormis que, à part que et à l'exception que permettent de signaler que l'idée exprimée dans la proposition qu'elles introduisent est opposée à celle exprimée dans la proposition antérieure. Au niveau de la syntaxe, ils affichent les mêmes propriétés que les conjonctions de coordination :
} 
(iii) Je suis la même personne, sauf que je me sens plus forte. (lemonde.fr)

(iv) *Sauf que je me sens plus forte, je suis la même personne.

(v) L'APL aiderait un quota résiduel de pauvres à se loger en HLM jusqu'à l'extinction définitive de la pauvreté. Sauf que la pauvreté et la précarité n'ont cessé d'augmenter en France depuis cette époque. (humanite.fr)

(vi) *L'APL aiderait un quota résiduel de pauvres à se loger en HLM jusqu'à l'extinction définitive de la pauvreté. Sauf que la pauvreté et sauf que la précarité n'ont cessé d'augmenter en France depuis cette époque.

L'étude de ces marqueurs dépasse le cadre de cet article.

${ }^{7}$ Un des relecteurs nous signale un cas de cooccurrence entre et et sauf trouvé dans Frantext :

(vii) C'est bien pourquoi sa machine de guerre va rouler sans accroc, chacun y trouvera son compte. Sauf les prochaines victimes de Paole, sauf Émile et sauf moi, qui vais sauter dans trois jours.

Un seul exemple ne suffit pas à invalider notre hypothèse. De plus, dans cet exemple, les syntagmes en sauf sont des compléments différés (après une ponctuation majeure). Or, on sait que de tels compléments forment des énoncés porteurs d'une valeur illocutoire propre. Il se peut donc que la conjonction et devant sauf moi ne marque pas la coordination de SE (au niveau microsyntaxique), mais plutôt une coordination d'assertions au niveau macrosyntaxique (cf. Benzitoun et al., 2010).

${ }^{8}$ Moignet $(1959$, p.11) parle de la mise à part : «On parlera de mise à part, par exemple, à propos de la phrase de Rabelais où il est dit (Gargantua, ch. XVII) que Gargantua " en noya deux cens soixante mille quatre cens diz et huit, sans les femmes et les petiz enfanz ". (Il n'est pas dit que les femmes et les petits enfants sont exceptés de la noyade, mais qu'ils n'entrent pas dans le compte des noyés). Si toute exception est une mise à part, toute mise à part n'est pas une exception. »

${ }^{9}$ Cela est d'ailleurs confirmé par des exemples authentiques :

(viii)Si l'on met à part le cas très particulier des titres britanniques, les performances 2016 sont clairement à l'avantage des Etats-Unis [...]. (lemonde.fr)

(ix) Si on met à part les CPI, un candidat issu d'un milieu social modeste va se tourner vers un IEJ. (nouvelobs.com)

(x) C'est tout ce qu'Amandine Buchard a ramené comme médaille d'or en 2017, si l'on excepte l'épreuve par équipe aux championnats d'Europe. (humanite.fr)

(xi) Cependant, si l'on excepte cette formule lapidaire, les propos du secrétaire d'Etat se sont révélés plus évasifs que volontaires. (lemoniteur.fr)

${ }^{10}$ Par contre, l'Académie Française met en garde contre ce mésusage : La locution prépositive sauf à peut signifier " sans que soit exclu le risque de », ou " en se réservant le droit de ». Elle ne signifie en aucun cas "sauf si » ou "à moins que ». Disponible à l'adresse suivante : http://www.academiefrancaise.fr/sauf.

${ }^{11}$ Cette analyse permet de privilégier le lien para entre les deux conjoints et de rendre compte de l'asymétrie de la construction (la conjonction forme un syntagme avec le conjoint qui suit et pas celui qui précède) (cf. Kahane et al., 2016. p. 26).

${ }^{12}$ Les données qui suivent nous ont été fournies par Marie-Amélie Botalla qui les a extraites de son corpus de thèse (Botalla, à paraitre).

${ }^{13}$ Universal Dependencies (UD) est un projet d'annotation syntaxique qui comprend plus de 100 treebanks dans plus de 70 langues, annotés avec le même schéma. Disponible à l'adresse suivante : universaldependencies.org.

${ }^{14}$ Un argument contre le classement des ME en anglais comme des prépositions a été fourni par García Álvarez, (2008), à la suite de Quinn (2005). Il a noté que les pronoms personnels suivis de butlexcept peuvent apparaître dans le cas accusatif aussi bien que dans le cas nominatif, un comportement possible avec les conjonctions de coordination, mais pas avec les prépositions (*with I).

(xii) Every defense attorney [Ep except I/me]. (García Álvarez, 2008, p.174)

Cet argument n'est pas applicable en français, puisque les conjonctions de coordinations peuvent seulement être suivies par un pronom fort $(*$ Pierre et je).

${ }^{15}$ Terme d'Abeillé et Mouret (2011) qui traduit gapping. 